\title{
MEJORAS EN LA DURABILIDAD DE VIGAS DE HORMIGÓN RECICLADO CON TRATAMIENTO SOMETIDAS A CARGAS EXTERNAS
}

\author{
R. S. MENESES ${ }^{1}$, J. M. MORO ${ }^{1}$, N. F. ORTEGA ${ }^{1,2}$ \\ ${ }^{1}$ Instituto de Ingeniería, Departamento de Ingeniería, Universidad Nacional Del Sur, CONICET \\ ${ }^{2}$ Instituto de Ingeniería, Departamento de Ingeniería, Universidad Nacional Del Sur, CIC.
}

\begin{abstract}
RESUMEN
La utilización de hormigones reciclados en estructuras resistentes es una alternativa de construcción sustentable. Sin embargo, este tipo de hormigones presenta características durables diferentes a aquellos elaborados en forma convencional, y por lo tanto afectan el comportamiento frente a ambientes agresivos de las estructuras en las que se utilizan dichos materiales. En este trabajo se presentan los resultados obtenidos al someter a un proceso de corrosión acelerada a vigas de hormigón armado, solicitadas a distintos esfuerzos flexionales. Se utilizaron hormigones reciclados con y sin tratamiento sobre los agregados de hormigón triturado. Se apreció la influencia positiva del tratamiento aplicado, desde el punto de vista durable. Por otra parte, se puso de manifiesto la influencia de la solicitación externa.
\end{abstract}

Palabras clave: Hormigón reciclado; Durabilidad; Estado de servicio; Tratamiento de agregados.

\begin{abstract}
The use of recycled concrete into resistant structures is a sustainable construction alternative. However, this type of concrete has different durability characteristics than those elaborated in conventional form. Therefore, affect the behavior against aggressive environments of the structures in which those materials are used. This paper presents, the results obtained to subjected to an accelerated corrosion process to reinforced concrete beams, applied to different flexural types. If they used recycled concrete with and without treatment on aggregates of crushed concrete. The positive influence of the applied treatment is appreciated from a durable point of view. On the other hand, the influence of the external request has been revealed.
\end{abstract}

Keywords: Recycled concrete; Durability, Service state, Aggregate treatments.

\section{RESUMO}

O uso de concreto reciclado em estruturas é uma alternativa de construção sustentável. No entanto, apresenta características de durabilidade diferentes do concreto convencional, características essas que afetam o comportamento da estrutura frente à ambientes agressivos. Neste trabalho, apresentam-se os resultados obtidos por aplicação de processo de corrosão acelerada em vigas de concreto armado, submetidas a diferentes esforços de flexão. Foram utilizados concretos reciclados com agregados de concreto triturado, com e sem tratamento. Foi possível observar a influência positiva da aplicação do tratamento nos agregados, do ponto de vista da durabilidade. Por outro lado, se pode observar a influência do tipo de carregamento no comportamento da viga.

Palavras-chave: Concreto reciclado; Durabilidade, Status do serviço; Tratamento de agregados. 


\section{INTRODUCCIÓN}

Un aspecto importante en el desempeño de las estructuras de hormigón armado es su durabilidad, la cual está vinculada con la estructura de poros del material y principalmente con la interconexión de ellos con el medio exterior (Zega, 2010). La corrosión de sus armaduras es una patología que puede afectar en gran medida a la durabilidad de estas estructuras y puede generar importantes daños que pueden derivar en el colapso estructural. La aparición de fisuras en el recubrimiento del hormigón, generadas por la acción expansiva de los productos de corrosión, que a su vez reducen la sección útil del hormigón (Andrade et al., 1996; Jin et al., 2015) es una característica propia de este proceso patológico, que sumado a la merma de la sección transversal de las armaduras y a la pérdida de la adherencia entre el hormigón y el acero (Ortega et al., 2001), hacen que disminuya el momento de inercia del elemento estructural, teniendo consecuencias negativas en la capacidad portante del mismo (Castaldo, 2017).

Las estructuras de hormigón armado en servicio, si sufren daños de corrosión en sus armaduras, generalmente, lo hacen bajo la acción de cargas externas, sin embargo, la mayoría de los artículos científicos que abordan este tema, se han centrado principalmente en las estructuras corroídas en ausencia de carga (Ortega et. al, 2001; Rodriguez et al., 1993; Almusallam et. al., 1996). El comportamiento que se espera de una estructura que está sometida a los efectos de la corrosión y en simultáneo bajo la acción de cargas externas, es diferente al de una estructura que a la que no se le aplican cargas, por este motivo las ejecuciones de algunas investigaciones fueron destinadas al estudio de esta temática, aunque son escasas en el contexto de la temática (Hariche et al., 2012; Malumbela et al, 2009; Yu et al., 2015). Con relación al estado tensional de las armaduras se puede decir que introduce una cierta incertidumbre en los procesos corrosivos y sus consecuencias. Pese a la importancia del tema que, en el caso de las armaduras sometidas a esfuerzos de tracción, se puede llegar a originar el fenómeno conocido como "corrosión bajo tensión", pero son escasas las investigaciones en estructuras de hormigón. No obstante, en los últimos años han aparecido algunos trabajos (Calabrese, 2013; Elfergani, 2012; Fumin, 2011; Ortega, 2011).

La utilización de elementos reciclados en la fabricación de materiales de construcción es una tendencia que se ha promovido notoriamente en los últimos años. Esto permite lograr ciclos de vida más prolongados y eficientes, en la generación de los mismos. Una alternativa para materializar esta iniciativa es, por ejemplo, el uso de agregados de hormigón triturado provenientes de la demolición de estructuras antiguas. En general, este tipo de agregados pueden presentar una alta porosidad, microfisuras, contenido de cloruros, sulfatos y otras impurezas, que afectan el rendimiento mecánico del hormigón reciclado (Ryu, 2003). Sin embargo, a pesar de presentar menor densidad y mayor absorción que los agregados naturales, es factible elaborar un hormigón de buen desempeño mediante una correcta dosificación (Lin et al., 2004; Evangelista et al., 2014).

La presencia de mortero adherido en la constitución de los agregados reciclados de hormigón implica una mayor porosidad de los mismos, frente a un agregado natural, esta característica hace que el hormigón obtenido con la utilización de este tipo de agregados presenta mayor porosidad que otro, de similares características, elaborado con agregados naturales. La capacidad y la velocidad de succión capilar se incrementan con el porcentaje de agregado reciclado (Villagrán et al., 2005). Todos estos motivos limitan el uso de agregados reciclados, por lo tanto, es importante analizar metodologías para mitigar sus debilidades.

En este trabajo se presentan resultados, que tienen como objetivo evaluar el comportamiento desde el punto de vista de la corrosión de sus armaduras y sus efectos externos (fisuración del recubrimiento del hormigón) e internos (potenciales de corrosión) de vigas de hormigón armado, sometidas a un proceso de corrosión acelerada y bajo la acción de cargas externas. En la fabricación de estas vigas, se empleó un hormigón en el que se reemplazó un porcentaje del agregado grueso natural por agregado reciclado 
de hormigón, el cual se utilizó sin someterse a ningún tratamiento y con a un tratamiento de lechada de cemento para disminuir su permeabilidad.

\section{MATERIALES Y MEZCLAS}

Se proyectaron diferentes hormigones reciclados de relación agua/cemento 0,50, con un $50 \%$ de reemplazo, en volumen, de agregado grueso natural (canto rodado) por agregado reciclado de hormigón. En la mitad de los casos, el agregado reciclado fue sometido a un tratamiento simultáneo al mezclado de los materiales mediante una lechada de cemento.

Los materiales utilizados en las mezclas fueron los siguientes:

Agua: se empleó agua corriente tomada de la red de provisión a la ciudad de Bahía Blanca.

Cemento: se usó cemento Portland normal (CPN 40) proveniente de una fábrica ubicada en la Provincia de Buenos Aires.

Agregado Fino: constituido por arena natural silícea proveniente de un yacimiento ubicado al sur de la Provincia de Buenos Aires, que cumple con las especificaciones de calidad dadas por la Norma IRAM 1627 (IRAM 1627, 1997).

Agregados gruesos, se emplearon dos tipos:

a) Reciclado: El hormigón a reciclar se obtuvo de la trituración de probetas ensayadas de hormigón elaborado con canto rodado, provenientes de distintas obras realizadas en la zona. Se molió el material a reciclar con el empleo de una trituradora de mandíbulas. El tamaño máximo nominal del material resultante fue de $25 \mathrm{~mm}$.

b) Reciclado con tratamiento: el material mencionado en el inciso a) fue sometido a un tratamiento con lechada de cemento.

En todos los casos, se mantuvo constante la relación agua/cemento (a/c) en 0,50, valor adoptado por la normativa argentina (CIRSOC 201, 2005), para estructuras resistentes que se encuentren a más de 1 $\mathrm{Km}$ de la costa, en zona influenciada por vientos con sales marinas.

\subsection{Elaboración de muestras}

Se fabricaron 8 vigas de hormigón armado con diferentes tipos de hormigones:

- Reciclado sin tratamiento previo (R): en su elaboración se reemplazó un 50\% del agregado natural por agregado triturado de hormigón.

- Reciclado con tratamiento previo (RT): en su elaboración se reemplazó un 50\% del agregado natural por agregado triturado de hormigón sometido a tratamiento previo de lechada de cemento.

También se moldearon probetas cilíndricas, con el objeto de caracterizar el comportamiento mecánico (compresión y tracción) y físico (ensayo de absorción capilar) de cada uno de los hormigones elaborados. (IRAM 1534, 2004; IRAM 1546, 1992; IRAM 1658, 1992; IRAM 1871, 2004)

\subsection{Tratamientos sobre el agregado reciclado}

La ejecución de este tratamiento se implementó sin la incorporación de otros materiales, modificando solamente el orden en la incorporación de materiales en la hormigonera. La carga de la misma se realizó en dos etapas; en la primera se colocó el agregado reciclado junto con una proporción de agua y cemento de la dosificación planteada, se mezcló durante 5 min., y luego se incorporó el resto de los materiales (agua, cemento, agregado grueso natural y agregado fino). Luego se mezclaron todos los materiales y se hormigonaron las muestras.

\subsection{Mezclas utilizadas}

La dosificación de los hormigones mencionados previamente, está detalladas en la Tabla 1, que representa la utilizada para los casos, ya sea con o sin tratamiento del agregado reciclado (R y RT). 
Tabla 1. Proporciones de la serie de mezclas realizadas con $\mathrm{a} / \mathrm{c}=0,50$

\begin{tabular}{|c|c|}
\hline Mezcla & 50\% A.R. \\
\hline Cemento $\left(\mathrm{kg} / \mathrm{m}^{3} \mathrm{H}^{\mathrm{o}}\right)$ & 350 \\
\hline Canto Rodado $\left(\mathrm{kg} / \mathrm{m}^{3} \mathrm{H}^{\mathrm{o}}\right)$ & 465 \\
\hline Agr. Reciclado $\left(\mathrm{kg} / \mathrm{m}^{3} \mathrm{H}^{\circ}\right)$ & 443 \\
\hline Agr. Fino $\left(\mathrm{kg} / \mathrm{m}^{3} \mathrm{H}^{\circ}\right)$ & 895 \\
\hline
\end{tabular}

En la Tabla 2 se muestran las dosificaciones utilizadas en cada etapa de mezclado para el hormigón tipo $\mathrm{RT}$, con el fin de realizar tratamiento sobre el agregado reciclado.

Tabla 2. Etapas de mezclado en el tratamiento del agregado reciclado (T)

\begin{tabular}{|c|c|c|}
\hline Etapa & Primera & Segunda \\
\hline Agua $\left(1 / \mathrm{m}^{3} \mathrm{H}^{\mathrm{o}}\right)$ & 87,5 & 87,5 \\
\hline Cemento $\left(\mathrm{kg} / \mathrm{m}^{3} \mathrm{H}^{\mathrm{o}}\right)$ & 175 & 175 \\
\hline Canto Rodado $\left(\mathrm{kg} / \mathrm{m}^{3} \mathrm{H}^{\mathrm{o}}\right)$ & 0 & 465 \\
\hline Agr. Reciclado $\left(\mathrm{kg} / \mathrm{m}^{3} \mathrm{H}^{\mathrm{o}}\right)$ & 443 & 0 \\
\hline Agr. Fino $\left(\mathrm{kg} / \mathrm{m}^{3} \mathrm{H}^{\mathrm{o}}\right)$ & 0 & 895 \\
\hline
\end{tabular}

\subsection{Características de las muestras}

Se realizaron ensayos en dos tipos de probetas:

a) Las vigas constaban de una sección rectangular de 8 x $16 \mathrm{~cm}$ y $220 \mathrm{~cm}$ de longitud, armadas longitudinalmente con barras de acero de dureza natural (IRAM-IAS U 500-528, 1989), de 4,2 mm de diámetro nominal (dos en el cordón superior y dos en el inferior), y estribos cerrados de acero liso de 2,1 $\mathrm{mm}$ de diámetro, separados cada $10 \mathrm{~cm}$, con un recubrimiento de $10 \mathrm{~mm}$. Estas, fueron usadas en los ensayos de corrosión acelerada. El llenado de los moldes se hizo en dos capas, compactándose mediante el uso de un vibrador y el curado se realizó en ambiente húmedo, durante 28 días en laboratorio, con las siguientes condiciones: $20 \pm 2{ }^{\circ} \mathrm{C}$ y HR $\approx 50 \%$.

b) Probetas cilíndricas de $10 \times 20 \mathrm{~cm}$ para realizar los ensayos físicos de capacidad y velocidad de succión capilar (IRAM 1871, 2004) y probetas cilíndricas de 15 x $30 \mathrm{~cm}$ para los ensayos mecánicos de resistencia a compresión (IRAM 1546, 1992) y tracción simple por compresión diametral (IRAM 1658, 1995). Se moldearon y curaron de acuerdo con la normativa vigente en nuestro país (IRAM 1534, 2004).

\subsection{Proceso de corrosión acelerada}

A los efectos de solicitar con esfuerzos de tracción, a las armaduras superiores de las vigas (que serían las que posteriormente serían sometidas a un proceso de corrosión acelerada), se colocaron cargas en sus extremos, siendo adoptada como zona a corroer una zona intermedia, dejando voladizos en los dos laterales. En la Figura 1, puede verse un esquema general de las vigas cargadas, durante el ensayo. 


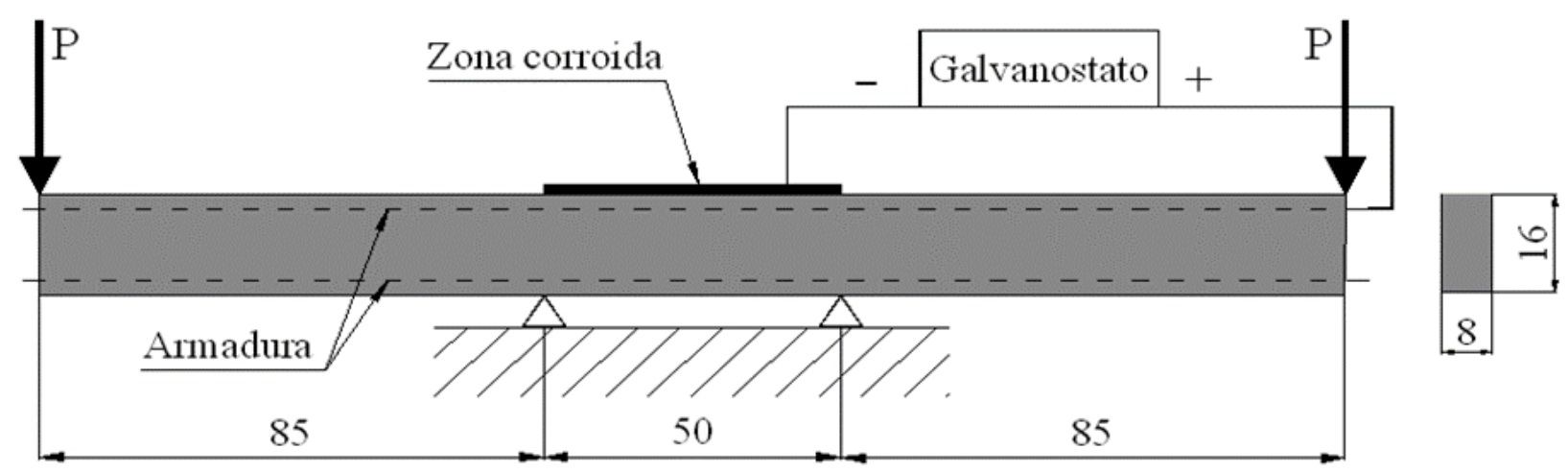

Figura 1: Esquema general del ensayo

Con el esquema estructural adoptado, la zona comprendida entre los apoyos, posee una solicitación a flexión aproximadamente constante, por lo que, toda la zona de las barras afectadas por la corrosión, está sometida a una tensión prácticamente constante, al estar expuestas, a su peso propio, más la acción de distintas cargas (P) que variaron desde $60 \mathrm{~kg}$ a $120 \mathrm{~kg}$.

Los elementos fueron sometidos a corrosión acelerada a lo largo de 120 días, mediante la aplicación de una corriente externa, provista por un galvanostato. Ésta fue aplicada a través de un contraelectrodo formado por una malla de acero inoxidable (de $50 \mathrm{~cm}$ de largo e igual ancho que la viga) ubicado sobre la superficie del hormigón en la parte superior de la viga. Sobre el mismo se dispuso una esponja (de iguales dimensiones), que se mantuvo humectada con una cantidad controlada de una solución de 0,3 $\%$ (en peso) de cloruro de sodio $(\mathrm{NaCl})$. Con el fin de asegurar que esa humectación fuera constante (para reducir la evaporación), se las cubrió con una placa de acrílico y luego una cobertura de nylon. La densidad de corriente aplicada fue de $100 \square \mathrm{A} / \mathrm{cm}^{2}$, que es alrededor de diez veces la medida en estructuras de hormigón armado altamente corroídas (Alonso et. al., 1998) fue elegida por haber sido adoptada en distintos trabajos sobre el tema (Alonso et. al., 1994; Aveldaño et. al., 2011). Con el objeto que al finalizar el ensayo se obtengan penetraciones de la corrosión superiores a 0,30 mm en las barras, sin producirse alteraciones significativas, respecto del proceso electroquímico que ocurre en una corrosión natural.

En la Tabla 3 se identifican las vigas ensayadas, de acuerdo al tipo de hormigón utilizado y a la carga aplicada en cada extremo (P).

Tabla 3. Denominación de las vigas

\begin{tabular}{|c|c|c|}
\hline Denominación & Tipo de Hormigón & $\begin{array}{c}\text { Carga aplicada (P) } \\
(\mathbf{K g})\end{array}$ \\
\hline VR60 & Reciclado & 60 \\
\hline VR80 & Reciclado & 80 \\
\hline VR90 & Reciclado & 100 \\
\hline VR100 & Reciclado & 120 \\
\hline VRT60 & Reciclado Tratado & 60 \\
\hline VRT80 & Reciclado Tratado & 80 \\
\hline VRT90 & Reciclado Tratado & 100 \\
\hline VRT100 & Reciclado Tratado & 120 \\
\hline
\end{tabular}




\subsection{Ensayos}

Los potenciales de corrosión, se midieron con un voltímetro CANIN (PROCEQ) que posee un electrodo de referencia de $\mathrm{Cu} / \mathrm{CuSO}_{4}$ (CSC) (ASTM C876, 1980). Primero, se efectuó la medición en seco para tener una referencia inicial del estado general de la viga, en los puntos que se iban a controlar a lo largo del ensayo. Luego se humectó durante dos días hasta alcanzar un estado de humectación constante con la solución de $\mathrm{NaCl}$ y posteriormente, se aplicó galvanostáticamente la densidad de corriente antes mencionada. Los resultados obtenidos se analizan en función de lo indicado por la norma (ASTM C87615, 2015).

Bajo estas condiciones, se siguió durante 120 días la evolución del potencial de corrosión, en diversos puntos característicos de la viga, sobre las zonas seca y húmeda sometidas a corrosión acelerada, en coincidencia o no con la ubicación de los estribos. Además, se efectuó un seguimiento del proceso de fisuración, detectando el momento de aparición de las primeras fisuras, para luego registrar periódicamente el largo y el ancho de las mismas. Las mediciones se realizaron con una escala graduada, cuya precisión era de $0,05 \mathrm{~mm}$, y su evolución se registró mediante gráficos, que muestran la forma en que discurrían.

\section{RESULTADOS Y DISCUSIÓN}

En la Tabla 4 se detallan los resultados obtenidos en los ensayos realizados para caracterizar las propiedades mecánicas de los hormigones elaborados y se incorporan los mismos parámetros determinados para un hormigón patrón, elaborado con la misma dosificación pero sin reemplazo de agregado natural por agregado reciclado (P) obtenidos en investigaciones previas (Moro et. al., 2017).

Tabla 4. Características mecánicas y físicas de los hormigones empleados.

\begin{tabular}{|c|c|c|}
\hline Probeta & $\begin{array}{c}\text { Compresión } \\
{[\mathbf{M P a}]}\end{array}$ & $\begin{array}{c}\text { Tracción Indirecta } \\
{[\mathbf{M P a}]}\end{array}$ \\
\hline $\mathrm{P}$ & 36,4 & 3,6 \\
\hline $\mathrm{R}$ & 32,6 & 2,8 \\
\hline $\mathrm{RT}$ & 33,3 & 3,3 \\
\hline
\end{tabular}

En la Tabla 5 se muestra la comparación porcentual de los valores de Capacidad de Succión Capilar (C) y de Velocidad de Succión Capilar (V), que se determinaron en el ensayo de absorción capilar de los hormigones elaborados. Tomando como referencia a los valores obtenidos en el hormigón patrón (P) mencionado previamente

Tabla 5: Características mecánicas y físicas de los hormigones empleados.

\begin{tabular}{|c|c|c|}
\hline Probeta & $\begin{array}{c}\text { C } \\
{[\%]}\end{array}$ & $\begin{array}{c}\text { V } \\
{[\%]}\end{array}$ \\
\hline P & 100 & 100 \\
\hline R & 176 & 152 \\
\hline RT & 103 & 102 \\
\hline
\end{tabular}

De estos resultados, se puede apreciar que aquellos hormigones elaborados con agregado grueso reciclado, a los que se les aplicó un tratamiento de lechada de cemento durante el mezclado, mostraron un leve incremento de su resistencia mecánica y una importante reducción de los valores de velocidad y capacidad de succión capilar, representando un comportamiento mecánico y de su estructura de poros similar al hormigón elaborado en forma convencional, sin la utilización de agregados reciclados (Moro et. al., 2017). 


\subsection{Potenciales de Corrosión}

En la Figura 2 se muestra el comportamiento electroquímico de las armaduras, mediante la representación de la evolución de los Potenciales de Corrosión en función de la Profundidad Media de Ataque sobre las barras metálicas, la cual se determinó, en forma teórica, a partir de la Ley de Faraday (Alonso et. Al., 1998), tomándose un promedio de los valores obtenidos para los 5 puntos, de la zona central afectada en cada una de las 6 vigas ensayadas.

\section{Profundidad Media de Ataque (mm)}

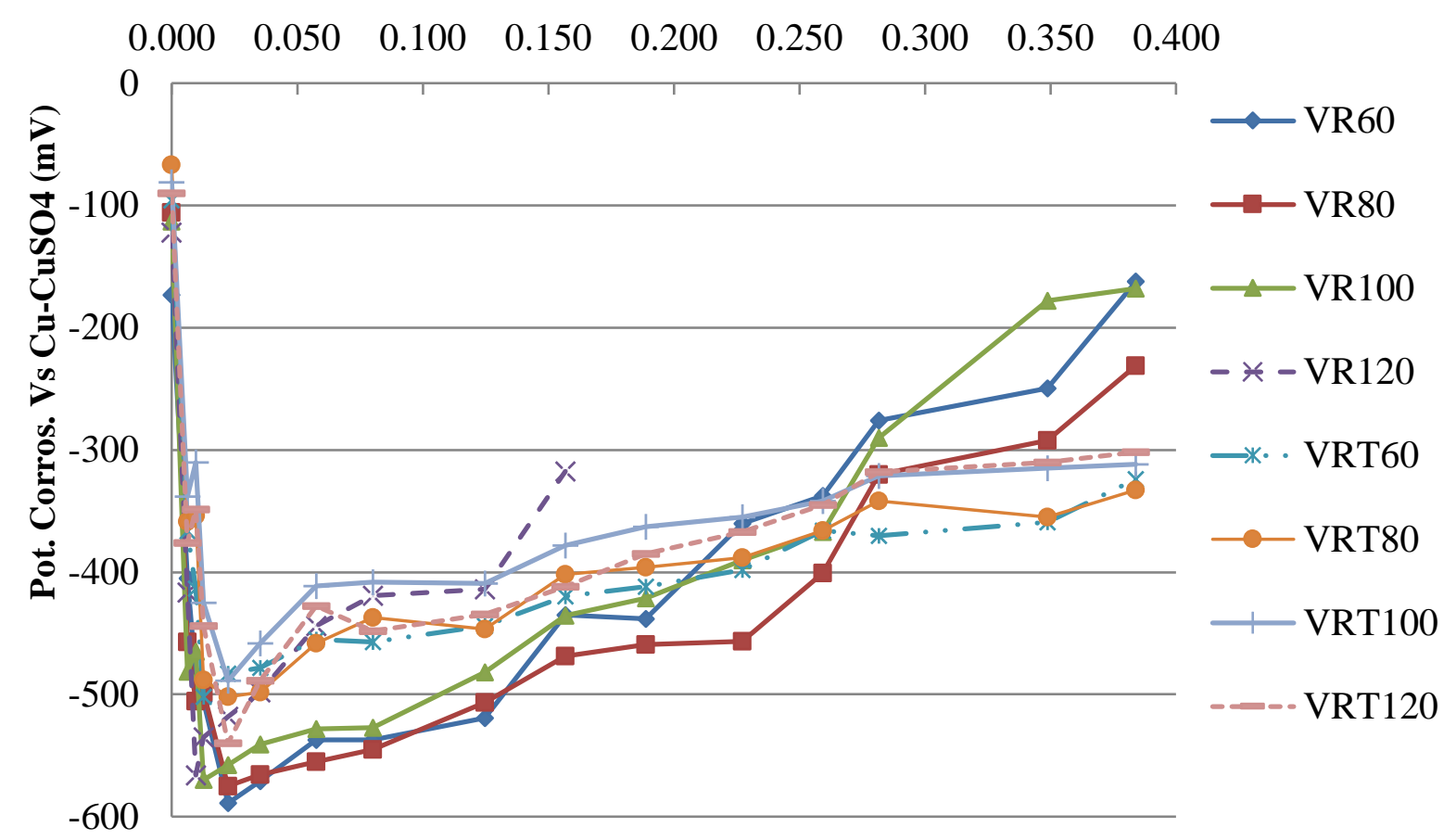

Figura 2. Potenciales de corrosión en función de la Prof. Media de Ataque a las armaduras.

Cabe acotar, que, si bien el ensayo se prolongó por aproximadamente 120 días, al arribar a los 51 días, la viga de hormigón reciclado sin tratamiento, sometida a mayor carga (VR120) llegó al colapso, debido a la debilitación de su armadura, por las picaduras generadas por la corrosión en las barras tracciondas. El comportamiento electroquímico analizado en las seis vigas, a través del seguimiento de los Potenciales de Corrosión, no mostró diferencias muy notables, observándose en todos los casos, el inicio del período de activación o despasivación de las armaduras, potenciales que no superaron los $590 \mathrm{mV}$. Posteriormente, se desarrolló un proceso de pseudo-pasivación y generalización de la fisuración, que fue similar en todas las vigas. Sin embargo, se puede observar que las vigas de hormigón reciclado sin tratamiento sobre los agregados $(\mathrm{R})$, presentaron mayores pendientes de pasivación que las elaboradas con agregados reciclados tratados (RT). Este comportamiento puede explicarse considerando que los hormigones cuyos agregados no fueron sometidos a tratamientos de impermeabilización, tienen una estructura de poros más abierta, permitiendo un mayor paso de humectación y oxígeno hacia las armaduras, y por lo tanto pasivándolas en forma más acelerada. Comparando este comportamiento con el observado en un estudio anterior realizado sobre vigas elaboradas con un hormigón de similar comportamiento mecánico que el utilizado en este trabajo, pero sin la incorporación de agregados reciclados (Ortega and Aveldaño, 2008), se puede apreciar que el período de activación de las armaduras, mostró sus picos de potencial en forma similar en ambos casos, tanto en las vigas con y sin fisuración por flexión. 


\subsection{Fisuración}

\subsubsection{Seguimiento de las áreas de fisuración}

Es sabido, que en estructuras de hormigón armado sometidas a flexión, cuando las tensiones en la zona de tracción, superan la resistencia del mismo, se generan fisuras que son denominadas "fisuras por flexión", identificables por su dirección transversal a la armadura principal de la viga. La presencia de estas fisuras hace que se acelere la penetración de oxígeno y cloruros hacia las armaduras, favoreciendo así el proceso de corrosión de las mismas (aumenta las áreas de fisuras por corrosión). Por otra parte, al ser las fisuras por flexión vías de egreso, de parte de los productos de corrosión, pueden llegar a crecer sus dimensiones.

Para las vigas utilizadas en este estudio, la carga de fisuración por flexión es de $85 \mathrm{~kg}$, por este motivo se trabajó con cargas inferiores $(60 \mathrm{~kg}$ y $80 \mathrm{~kg}$ ) y superiores $(100 \mathrm{~kg}$ y $120 \mathrm{~kg})$ a la misma, para poder analizar el comportamiento de las mismas, con diferentes estados tensionales de sus armaduras y de fisuración inicial.

En este trabajo se muestran las Áreas de Fisuración Totales, es decir las obtenidas mediante la suma de las áreas de fisuración por flexión, más las de fisuración por corrosión. Cabe aclarar, que se denomina Área de Fisuración al producto de la longitud y el ancho de cada fisura. En la Figura 3 se aprecia el avance del Área de Fisuración Total de cada viga, en función del tiempo.

Se observa que las vigas VR60, VR80 muestran una variación similar en el incremento del Área de Fisuración Total en el tiempo, mientras que las vigas VR100 y VR120 cuyas cargas hacen que se supere la tensión de fisuración en el hormigón, poseen un comportamiento diferenciado frente a las anteriores, debido a la influencia que tiene esta fisuración, provocando una variación apreciable del incremento del Área de Fisuración Total en el tiempo. Similar comportamiento también se manifiesta en las vigas VRT60 y VRT80 respecto a las vigas VRT100 y VRT120, pero siempre con pendientes superiores, considerando si se considera la misma magnitud de carga externa.

Por otro lado, se puede apreciar que las pendientes de las curvas correspondientes a las Áreas de Fisuración son superiores para las vigas de hormigón reciclado en las que se usaron agregados reciclados tratados RT. Esto se debe a que, al presentar una estructura de poros más cerrada, una vez que se inicia el proceso de corrosión, los productos generados por el mismo, cuentan con menor espacio para alojarse, generando mayores tensiones en el hormigón circundante, y por lo tanto, mayor fisuración.

En la Figura 4 se muestra la variación del Ancho Máximo de Fisuras, en función de la Profundidad Media de Ataque a las armaduras, y en la Figura 5 se discrimina la variación del Ancho Máximo de Fisuras ocasionadas únicamente por flexión.

Se aprecia, al igual que en las Áreas de Fisuración, que en las vigas sometidas a cargas superiores existe una tendencia a mayor crecimiento en los Anchos Máximos de Fisuras en el tiempo. En este sentido, se puede apreciar en las Tabla 6, las pendientes en el Análisis de Regresión realizado indicaron un crecimiento mayor de estos parámetros en las vigas tipo RT, las cuales fueron elaboradas con hormigones que mostraron mayor resistencia mecánica y menor absorción capilar que el restante, considerando apta la justificación de este fenómeno expresado para la las Áreas de Fisuración. 


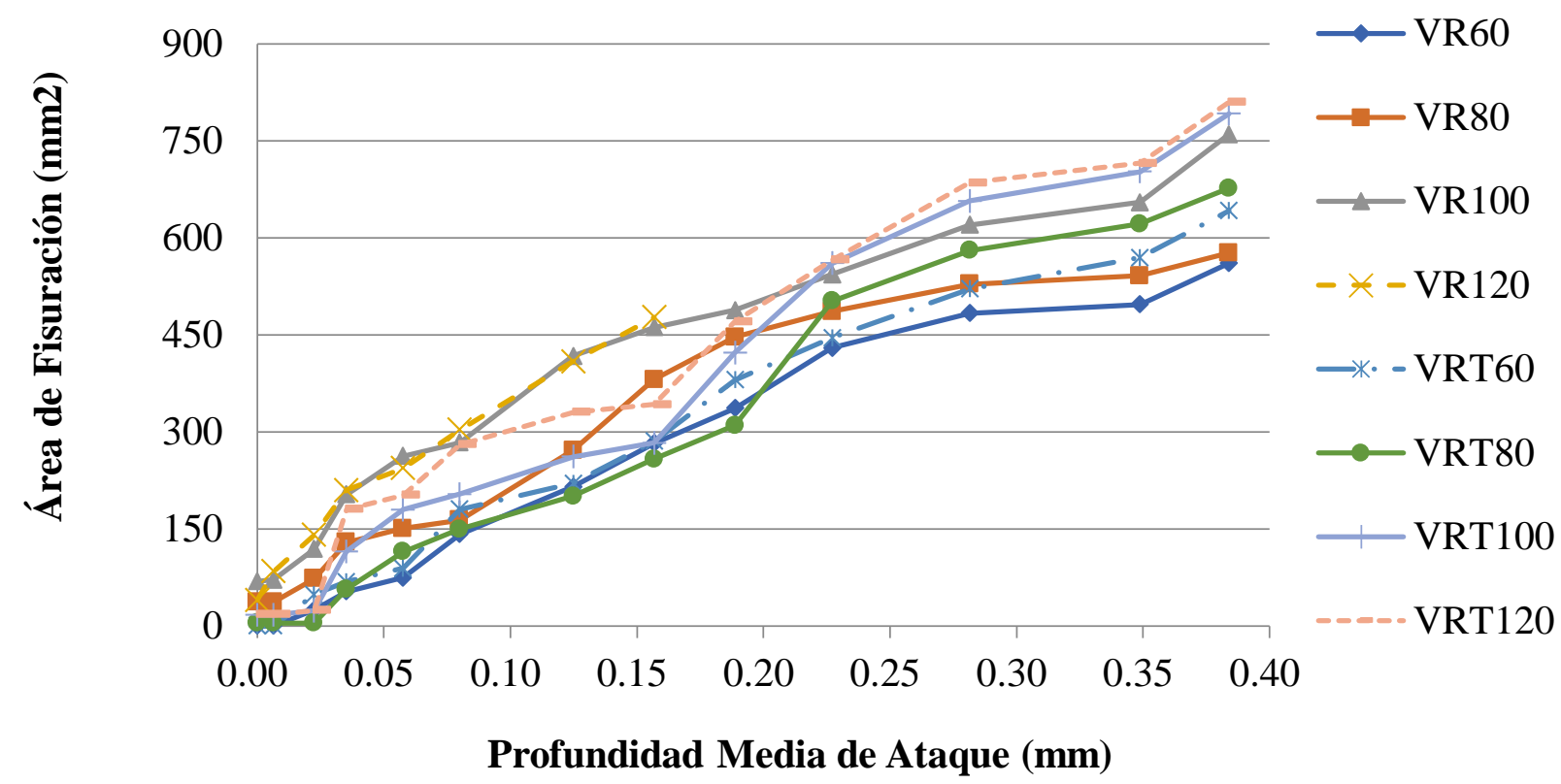

Figura 3. Área de Fisuración en función de la Profundidad Media de Ataque a las armaduras.

Por su parte, los anchos Máximos de Fisuras por flexión, como era de esperar fue superior en las vigas que soportaban mayores cargas, sin embargo, la viga VR120 presentó valores muy superiores al resto; esto se aprecia claramente con el estado al que llegó de dicha viga, donde el deterioro por corrosión se dio en mayor medida en la zona de las fisuras por flexión, llegando al colapso a temprana edad.

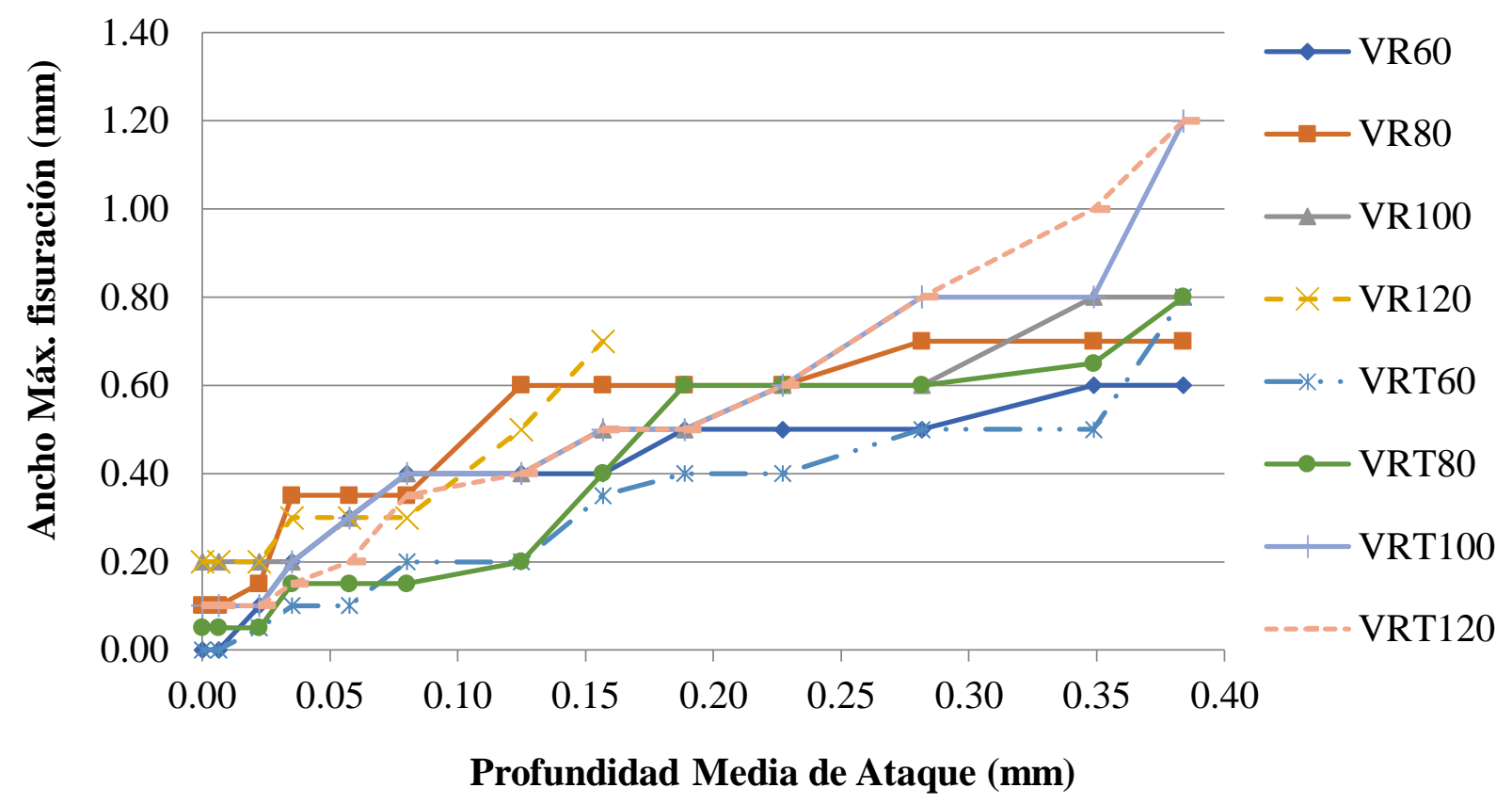

Figura 4: Ancho Máximo de Fisuración en función de la Prof. Media de Ataque a las armaduras. 


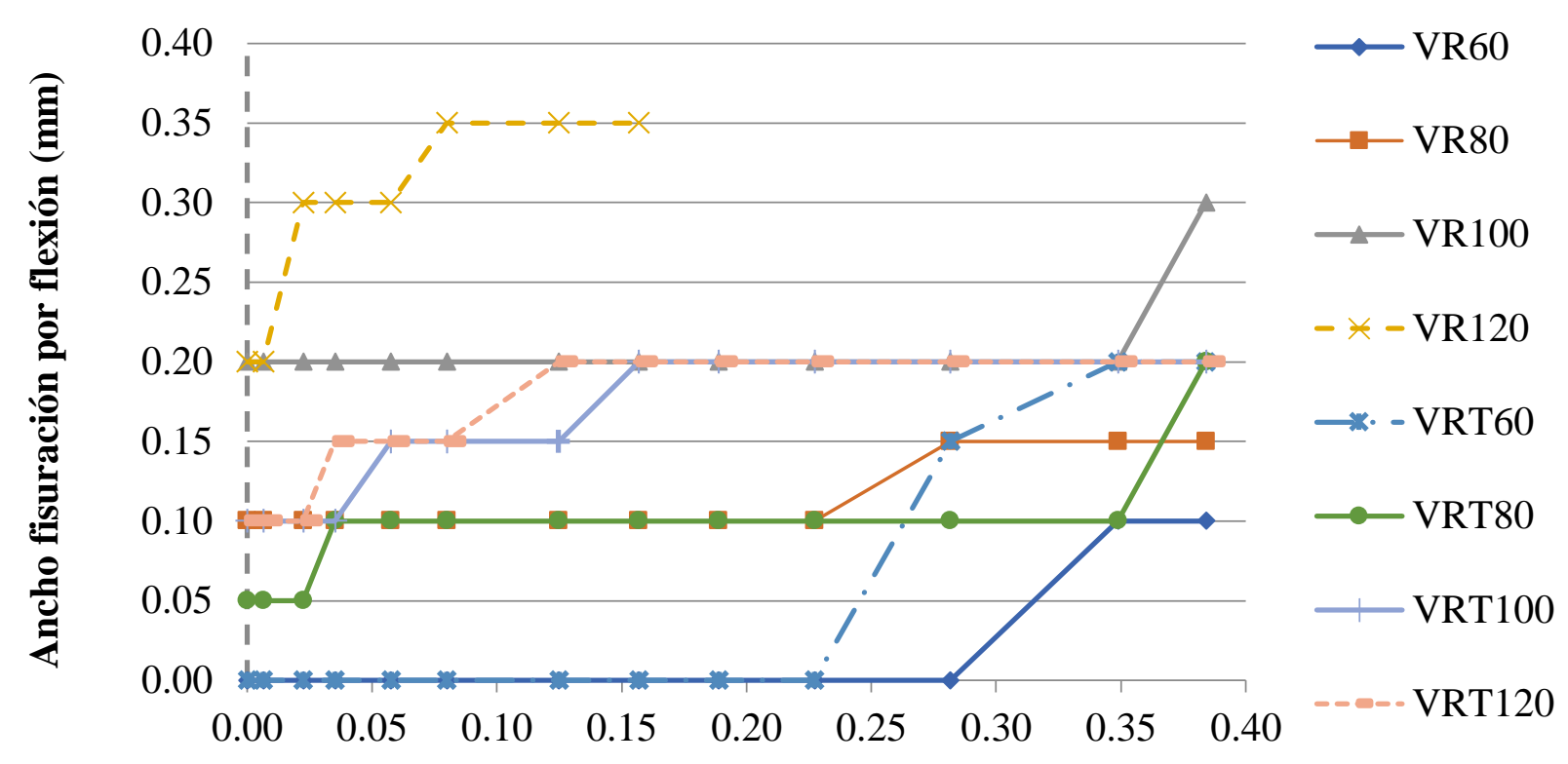

\section{Profundidad Media de Ataque (mm)}

Figura 5: Ancho Máx. de Fisuración por flexión en función de la Profundidad Media de Ataque a las armaduras.

Tabla 6: Variación de Área de Fisuración (y), respecto a Profundidad Media de Ataque (x) y Variación de los Anchos Máximos de Fisuras (y), respecto a la Profundidad Media de Ataque (x)

\begin{tabular}{|l|c|c|c|c|}
\hline \multirow{2}{*}{ Viga } & \multicolumn{2}{|c|}{ Áreas de fisuración } & \multicolumn{2}{c|}{ Ancho Máximo de fisuración } \\
\cline { 2 - 6 } & Ley & $\begin{array}{c}\text { Índice de } \\
\text { corrección }\end{array}$ & Ley & $\begin{array}{c}\text { Índice de } \\
\text { corrección }\end{array}$ \\
\hline VR60 & $\mathrm{y}=4,9632 \mathrm{x}+10.288$ & 0,8073 & $\mathrm{y}=0,0046 \mathrm{x}+0,1334$ & 0,8073 \\
\hline VR80 & $\mathrm{y}=4,8696 \mathrm{x}+70,212$ & 0,7978 & $\mathrm{y}=0,0051 \mathrm{x}+0,2215$ & 0,7978 \\
\hline VR100 & $\mathrm{y}=5,5057 \mathrm{x}+127.96$ & 0,9691 & $\mathrm{y}=0,0052 \mathrm{x}+0,1971$ & 0,9691 \\
\hline VR120 & $\mathrm{y}=8,5363 \mathrm{x}+77.540$ & 0,9493 & $\mathrm{y}=0,0095 \mathrm{x}+0,1593$ & 0,9493 \\
\hline VRT60 & $\mathrm{y}=5,4822 \mathrm{x}+13.380$ & 0,9848 & $\mathrm{y}=0,0057 \mathrm{x}+0,0156$ & 0,9420 \\
\hline VRT80 & $\mathrm{y}=6,0070 \mathrm{x}+8.1658$ & 0,9775 & $\mathrm{y}=0,0064 \mathrm{x}+0,0471$ & 0,9251 \\
\hline VRT100 & $\mathrm{y}=6,6309 \mathrm{x}+22.880$ & 0,9792 & $\mathrm{y}=0,0078 \mathrm{x}+0,1036$ & 0,9424 \\
\hline VRT120 & $\mathrm{y}=6,5890 \mathrm{x}+54.133$ & 0,9684 & $\mathrm{y}=0,0087 \mathrm{x}+0,0619$ & 0,9808 \\
\hline
\end{tabular}

De la inspección visual de las armaduras descubiertas una vez finalizado el ensayo, se puede ver que, debido a la acción de los Cloruros, la corrosión sobre las barras no fue solamente generalizada, sino que se detectaron zonas de corrosión localizada (picaduras) a pesar de la baja concentración adoptada en la solución de humectación. Además, se observó corrosión de la parte superior de los estribos que hizo que algunos sectores de estribos prácticamente desaparecieran, no obstante, no se vieron afectadas las barras inferiores. 


\section{CONCLUSIONES}

Los hormigones elaborados, con reemplazó del 50\% del agregado grueso natural, por agregado reciclado de hormigón, al cual se le aplicó un tratamiento de lechada de cemento, mostraron una clara reducción en su estructura de poros, respecto al hormigón reciclado sin tratamiento, presentando un comportamiento mecánico y de absorción capilar similar al hormigón tradicional, realizado sin utilización de agregado reciclado.

El comportamiento frente al proceso de corrosión de las armaduras que presentaron las vigas elaboradas con hormigón reciclado con tratamiento (RT) mostró un mayor Ancho Máximo de Fisuras y Área de Fisuración que las vigas elaboradas con hormigones reciclados sin tratamientos, los cuales presentaban una estructura de poros más abierta.

Las fisuras por corrosión que se generan en las vigas cargadas, las cuales presentan fisuras por flexión, avanzan con mayor rapidez, que en los casos que no se aplican cargas externas y en los casos que las cargas no provocan fisuras por flexión. Además, existe una relación directa entre el incremento de la fisuración y el aumento de la carga aplicada, marcándose esta diferencia cuando la carga sobrepasa la carga de fisuración.; evidentemente las fisuras por flexión son una vía de ingreso de las sustancias que favorecen el proceso de corrosión.

El estudio del comportamiento de vigas de hormigón reciclado frente a procesos de corrosión, y además, bajo la acción de cargas, permite analizar la posible aplicación de este tipo de hormigones en estructuras resistentes.

La utilización de tratamientos aplicados sobre el agregado reciclado de hormigón, es una alternativa amigable con el ambiente. Estos permiten la disminución de la absorción capilar del hormigón, obtenido utilizando al mismo como parte del agregado grueso, de esta manera se mejora la durabilidad de la estructura.

\section{AGRADECIMIENTOS}

Los autores desean expresar su agradecimiento a la Dra. Ing. Carla Priano, Ing. Lilian Señas y a los técnicos Juan P. Gorordo del Laboratorio de Modelos Estructurales y Diego Smith del Laboratorio de Estudio y Ensayos de Materiales, U.N.S., por la amplia colaboración prestada para la elaboración de los ensayos de este trabajo. Además, se agradece al CONICET, a las Secretarías de Ciencia y Tecnología de la Universidad Nacional del Sur y al Departamento de Ingeniería por su aporte económico e institucional necesarios para la materialización de estas investigaciones.

\section{REFERENCIAS}

American Society for Testing and Materials (2015), ASTM C876-15, "Standard Test Method for Half Cell Potencial of Reinforcing Steel in Concrete", Philadelphia, U.S.A..

Almusallam, A. A., Al-Gahtani A. S., Rasheeduzzafar A. Z. A., (1996), "Effect of reinforcement corrosion on bond strength", Construction and Building Materials, n. ${ }^{\circ} 10,2$, pp. 123-129. https://doi.org/10.1016/0950-0618(95)00077.

Alonso C., Andrade C., Rodriguez J., Diez J. M., (1998), "Factors controlling cracking in concrete affected by reinforcement corrosión”, Materials and Structures, n. ${ }^{\circ 31}$, pp. 435-445.

Alonso M.C., Andrade M.C., Rodríguez J., Casal J., García A.M., (1994), “Evaluación experimental de la fisuración del hormigón producida por la corrosión de las armaduras”, Hormigón y Acero $\mathrm{N}^{\circ} 194$, pp. 29-42. 
Andrade, C., Alonso, C., Rodriguez, J., \& Garcia, M., (1996)., "Cover cracking and amount of rebar corrosion: importance of the current applied accelerated tests. In concrete repair, Rehabilitation and protection”, London, UK, RK Dhir and MR Jones, E\&FN Spon pp. 263-273.

Aveldaño, R. R., Ortega, N. F., (2009), "Influence of reinforcement distribution in the corrosive process of reinforced concrete beam", Magazine of Concrete Research, Thomas Telford, V. 61, Nr. 3, pp. 213220. https://doi.org/10.1680/macr.2008.00013.

Aveldaño, R. R., Ortega, N. F. (2011), "Characterization of Concrete Cracking due to Corrosion of Reinforcements in Different Environments", Construction and Building Materials,V.25, pp.630-37. https://doi.org/10.1016/j.conbuildmat.2010.07.029

Aveldaño, R. R., Ortega, N. F., (2013), "Behavior of concrete elements subjected to corrosion in their compressed or tensed reinforcement, Construction and Building Materials, Nr. 38, pp. 822-828. https://doi.org/10.1016/j.conbuildmat.2012.09.039.

Calabrese, L., Campanella, G., Proverbio, E., (2013), "Identification of corrosion mechanisms by univariate and multivariate statistical analysis during long term acoustic emission monitoring on a prestressed concrete beam", Corrosion Science, V. 73, pp. 161-171. https://doi.org/10.1016/j.corsci.2013.03.032

Castaldo, P., Palazzo, B., Mariniello, A., (2017), "Effects of the axial force eccentricity on the timevariant structural reliability of aging rc cross-sections subjected to chloride-induced corrosion", Engineering Structures, 130, pp. 261-274. https://doi.org/10.1016/j.engstruct.2016.10.053.

Elfergani, H. A., Rhys, P., Holford, K. M., (2013), "Damage assessment of corrosion in prestressed concrete by acoustic emission", Construction and Building Materials, V. 40, pp. 925-933. https://doi.org/10.1016/j.conbuildmat.2012.11.071

Fumin, L., Yingshu, Y., Chun-Qing, L., (2011), "Corrosion propagation of prestressing steel strands in concrete subject to chloride attack", Construction and Building Materials, V. 25, Nr.10, pp. 38783885. https://doi.org/10.1016/j.conbuildmat.2011.04.011

Hariche, L., Ballim, Y., Bouhicha, M. Kenai, S., (2012), "Effects of reinforcement configuration and sustained load on the behaviour of reinforced concrete beams affected by reinforcing steel corrosion", Cement and Concrete Composites, V. 34, Nr. 10, pp. 1202-1209. https://doi.org/10.1016/j.cemconcomp.2012.07.010

CIRSOC: Reglamento 201, (2005), "Proyecto, Cálculo y Ejecución de Estructuras de Hormigón Armado y Pretensado", 2, Bs. As.

Evangelista, L., De Brito, J., (2014), “Concrete with fine recycled aggregates: a review.”, European Journal of Environmental and Civil Engineering, 18(2), pp. 129-72. https://doi.org/10.1080/19648189.2013.851038

Instituto Argentino de Racionalización de Materiales - Instituto Argentino de Siderurgia, (1989), "Norma U 500-528: Barras de acero conformadas de dureza natural, para armadura en estructuras de hormigón”, Buenos Aires.

Instituto Argentino de Racionalización de Materiales 1627, (1997). “Agregados. Granulometría de los agregados para hormigón”. IRAM, Buenos Aires.

Instituto Argentino de Racionalización de Materiales 1534, (2004), "Hormigón. Preparación y curado de probetas en laboratorio para ensayos de compresión y tracción por compresión diametral.",IRAM, Bs. As.

Instituto Argentino de Racionalización de Materiales 1546, (1992). "Hormigón de cemento portland. Método de ensayo de compresión.",IRAM, Bs. As.

Instituto Argentino de Racionalización de Materiales 1658, (1995). "Hormigón. Método de ensayo de tracción simple por compresión diametral.", IRAM, Bs. As.

Instituto Argentino de Racionalización de Materiales 1871, (2004). "Método de ensayo para determinar la capacidad y la velocidad de succión capilar de agua del hormigón endurecido.", IRAM, Bs. As. 
Jin, L., Zhang R., Du X., Li, Y., (2015), “Investigation on the cracking behavior of concrete cover induced by corner located rebar corrosion. Engineering Failure Analysis, 52, pp. 129-143. https://doi.org/10.1016/j.engfailanal.2015.03.019

Lin, Y., Tyan, Y., Chang, T. \& Chang, C. (2004), An assessment of optimal mixture for concrete made with recycled concrete aggregates.", Cement and Concrete Research, 34, pp. 1373-80. https://doi.org/10.1016/j.cemconres.2003.12.032

Malumbela, G., Moyo, P., Alexander, M., (2009), "Behavior of RC beams under sustained service loads.", Construction and Building Materials, V. 23, Nr. 11, pp. 3346-3351. https://doi.org/10.1016/j.conbuildmat.2009.06.005

Moro J. M., Meneses R.S., Ortega N.F., Aveldaño R.R., (2017), "Variantes para mejorar el comportamiento ante procesos de corrosión de elementos de hormigón reciclado”, XIV Congreso latino-americano de patología de construcción - XVI Congreso de control de calidad en la construcción. Ortega, N., Alonso, M., Andrade, M. López, C., (2001), “Análisis de la fisuración ocasionada por la corrosión de las armaduras activas de elementos pretensados", Proceedings of Coloquia, 10.

Ortega N.F., Aveldaño R.R., (2008), "Analysis of tensed reinforced concrete beams during the corrosion process. ", Construction and Building Technology Journal, Bentham OPEN (Bentham

Science Publishers Ltd.), Vol. 2, pp. 243-250.

Rodríguez J., Ortega L.M., Casal J., Vidal M.A., (1993), "Disminución de la adherencia entre hormigón y barras corrugadas debido a la corrosión.”, Hormigón y Acero, 189, pp. 49-65.

Ryu, J.S., (2003), "An experimental study on the effect of recycled aggregate on concrete properties.", Magazine of Concrete Research, 54(1), pp. 7-12. https://doi.org/10.1680/macr.2002.54.1.7

Villagrán Zacardi, Y., Taus, V., Zega, C. Di Maio, A., Traversa, L., (2005). "Propiedades de transporte en hormigones convencionales y reciclados y su influencia en la corrosión de armaduras.", Simposio Internacional fib "El Hormigón Estructural y el Transcurso del Tiempo". La Plata, 1, pp. 91-8.

Yu, L., François, R., Dang, V. H., L'Hostis, V., Gagné, R., (2015), “Development of chloride-induced corrosion in pre-cracked RC beams under sustained loading: Effect of load-induced cracks, concrete cover, and exposure conditions", Cement \& Concrete Research, N67, 2015, pp.246-258. https://doi.org/10.1016/j.cemconres.2014.10.007.

Zega, C., (2010), "Propiedades físico-mecánicas y durables de hormigones reciclados.", Tesis de doctorado inédita, Universidad Nacional de La Plata. Facultad de Ingeniería, La Plata. 